Revue d'histoire de l'Amérique française

REVUE D'HISTOIRE DE L'AMÉRIQUE FRANÇAISE

\title{
La bibliothèque de l'Institut canadien de Montréal (1852-1876) : pour une analyse multidimensionnelle
}

\section{Yvan Lamonde}

Volume 41, numéro 3, hiver 1988

URI : https://id.erudit.org/iderudit/304582ar

DOI : https://doi.org/10.7202/304582ar

Aller au sommaire du numéro

Éditeur(s)

Institut d'histoire de l'Amérique française

ISSN

0035-2357 (imprimé)

1492-1383 (numérique)

Découvrir la revue

Citer cet article

Lamonde, Y. (1988). La bibliothèque de l'Institut canadien de Montréal (1852-1876) : pour une analyse multidimensionnelle. Revue d'histoire de l'Amérique française, 41(3), 335-361. https://doi.org/10.7202/304582ar
Résumé de l'article

La préoccupation méthodologique domine l'étude historique qui suit. À partir d'une " série " documentaire - des catalogues et des registres d'emprunt -, on propose une méthode d'analyse de ces documents, une méthode de classification qui n'existait pas, au plan international, pour l'étude des bibliothèques du $19^{\mathrm{e}}$ siècle. Suit une analyse quantitative de la collection et de la circulation des livres à l'Institut Canadien, puis une analyse qualitative qui reporte le contenu de la bibliothèque sur l'ensemble des activités de l'Institut. Les conclusions proposent de revoir certains aspects de l'histoire de l'Institut Canadien de Montréal. 


\title{
LA BIBLIOTHÈQUE DE L'INSTITUT CANADIEN DE MONTRÉAL (1852-1876): POUR UNE ANALYSE MULTIDIMENSIONNELLE
}

YVAN LAMONDE

Université McGill

\begin{abstract}
RÉSUMÉ
La préoccupation méthodologique domine l'étude historique qui suit. À partir d'une «série» documentaire - des catalogues et des registres d'emprunt - on propose une méthode d'analyse de ces documents, une méthode de classification qui n'existait pas, au plan international, pour l'étude des bibliothèques du $19 \mathrm{e}$ siècle. Suit une analyse quantitative de la collection $e t$ de la circulation des livres à l'Institut Canadien, puis une analyse qualitative qui reporte le contenu de la bibliothèque sur l'ensemble des activités de l'Institut. Les conclusions proposent de revoir certains aspects de l'histoire de l'Institut Canadien de Montréal.
\end{abstract}

\section{ABSTRACT}

The following study focusses on methodological solutions. A method is proposed to analyse «series» of documents - library catalogues and loan registers -, such a methodology for studying libraries and bookstores contents was lacking at an international level. Applied, this method allows a quantitative analysis of both the collection and circulation of books at the Institut Canadien de Montréal. The quantitative analysis is completed by a qualitative analysis which puts the library in the context of the other activities of the Institut. Conclusions proposed to review some aspects of the Institut Canadien's history.

La recherche sur l'histoire de l'imprimé achève d'enfoncer le mythe selon lequel cette histoire «livresque» ne serait que l'envers élitiste d'une pratique historienne elle-même consommatrice de livres et d'imprimés. Les travaux en histoire socio-culturelle ici et ailleurs ont maintenant fait la preuve que les formes culturelles populaires et bourgeoises, orales et écrites, empruntent de singuliers détours et entretiennent d'inattendues relations.

Tant que cette histoire de l'imprimé ne s'était pas donné des moyens analytiques valables, elle demeurait qualitative, impressionniste, s'offrant le luxe des généralisations abusives. Mais le temps des méthodes est venu. Et du coup celui aussi de la découverte des potentialités d'une telle histoire.

La présente étude propose et applique une méthode d'analyse du contenu de bibliothèques, abordant ici monographiquement le cas de 
l'Institut Canadien de Montréal. Non seulement prétend-elle solutionner le problème méthodologique de l'analyse des catalogues et de la classification des titres, mais surtout elle double une analyse quantitative de la collection et de la circulation d'une analyse qualitative qui reporte le contenu de la bibliothèque sur l'ensemble des activités de l'Institut Canadien et qui projette un éclairage nouveau sur le courant libéral et sur la censure épiscopale et romaine.

L'analyse de catalogues de bibliothèques de collectivités ne peut révéler qu'une chose: l'état d'une collection, l'offre de volumes, leur disponibilité en un lieu et à un temps donnés. L'analyse des registres d'emprunts permet d'étudier la circulation et de supposer la lecture possible ou probable des volumes empruntés. Mais la conservation des registres d'emprunt étant exceptionnelle, l'analyse du catalogue et de la collection s'imposera toujours.

Les trois catalogues de la bibliothèque de l'Institut Canadien de Montréal étudiés ici - ceux de 1852, de 1870 et le Supplément de $1876^{1}$ — permettent l'analyse, sur plus d'un quart de siècle, du contenu de cette bibliothèque qui ouvre ses portes en 1845 et les ferme en $1880^{2}$.

Leur mode de présentation pose du coup tout le problème méthodologique de l'analyse des catalogues de bibliothèques au 19e siècle. Alors que le Catalogue de 1852 se présente tout simplement selon un ordre alphabétique de titres avec mention de l'auteur et du nombre de volumes, ceux de 1870 et de 1876 décrivent, sous douze «sections» ou rubriques de classification, les titres par ordre alphabétique, avec mention de l'auteur, du nombre de volumes et d'une cote de localisation des ouvrages dans la bibliothèque (tableau 1 ).

1 Catalogue de la bibliothèque de l'Institut Canadien (Montréal, Imprimé par W.-H. Bowen, février 1852), 36 p.; Catalogue des livres de la bibliothèque de l'Institut Canadien (Montréal, Imprimerie de Alphonse Doutre et Cie, 1870), 47 p., classé par ordre de matière et arrangé alphabétiquement par A. Boisseau; Catalogue des livres de la bibliothèque de l'Institut Canadien, Supplément no 1 (Montréal, Imprimerie de Alphonse Doutre et Cie, 1876), 13 p.; malgré la datation de 1870 en page couverture (1876 en page 1), ce Supplément est bien de 1876: en témoignent les titres sur l'affaire Guibord, l'Histoire de la littérature canadienne (1874) de Lareau, les Chroniques, humeurs et caprices (1873) de Buies et Le Canada sous l'Union (1871-1872) de L.-P. Turcotte.

2 Yvan Lamonde, Les bibliothèques de collectivités à Montréal (17e-l9e siècle). Sources et problèmes (Montréal, Bibliothèque nationale du Québec, 1979), 53-60; «Histoire et inventaire des archives de l'Institut Canadien de Montréal (1855-1900)», Revue d'histoire de l'Amérique française, 28,1 (juin 1974): 77-93. Armande Lebeau a déjà étudié la collection littéraire dans L'Institut Canadien de Montréal (1844-1883). Le contenu littéraire de la bibliothèque, thèse de M.A. (littérature), Université McGill, 1981, iv-81 p.; Louis-Georges Harvey et Mark Olsen ont, par analyse informatique, étudié la collection, la circulation et les titres «à l'Index»: L.-G. Harvey, Étude de la bibliothèque de l'Institut Canadien de Montréal, thèse de M.A. (histoire), Universitê d'Ottawa, 1982; L.-G. Harvey and Mark Olsen, «A Quantitative Study of Book Circulation: the Library of the Institut Canadien de Montréal», Historical Methods, 18,3 (1985): 97-103; «La circulation de la bibliothèque de l'Institut Canadien de Montréal, 1865-1875», à paraître dans Histoire sociale/Social History. 
TABLEAU 1

Institut Canadien de Montréal, «sections» du Catalogue (1870 et Supplément 1876) de la bibliothèque, nombre de titres par section et $\%$.

\begin{tabular}{|c|c|c|c|c|c|c|c|}
\hline & & 187 & & Supp & & TOT & \\
\hline & & $\mathrm{N}$ & $\%$ & $\mathrm{~N}$ & $\%$ & $\mathrm{~N}$ & $\%$ \\
\hline A & $\begin{array}{l}\text { : Philosophie, religion, économie } \\
\text { politique, etc... }\end{array}$ & 212 & 11,4 & 56 & 9,3 & 268 & 10,9 \\
\hline B.C.D. & $\begin{array}{l}\text { : Romans modernes et anciens et } \\
\text { autres ouvrages }\end{array}$ & 654 & 35,3 & 285 & 47,6 & 939 & 38,3 \\
\hline E.F. & $\begin{array}{l}\text { : Littérature, auteurs classiques, } \\
\text { poésie, théâtre, etc... }\end{array}$ & 187 & 10 & 81 & 13,5 & 268 & 10,9 \\
\hline G.H.I. & : Livres de loi, statuts, etc... & 51 & 2,7 & 42 & 7 & 93 & 3,8 \\
\hline J.K. & $\begin{array}{l}\text { Correspondance de Napoléon } \\
\text { ler, comptes rendus Académie } \\
\text { des sciences, dictionnaires, } \\
\text { etc... }\end{array}$ & 32 & 1,7 & - & & 32 & 1,3 \\
\hline $\mathrm{L}$ & $\begin{array}{l}\text { : Dons du prince Napoléon, gra- } \\
\text { vures, cartes, plans, etc. }\end{array}$ & 60 & 3,2 & - & & 60 & 2,4 \\
\hline M & $\begin{array}{l}\text { : Livres d'écoles, dictionnaires, } \\
\text { gravures, etc. }\end{array}$ & 150 & 8 & - & & 150 & 6,1 \\
\hline N.O. & $\begin{array}{l}\text { : Histoire, mémoires historiques, } \\
\text { etc... }\end{array}$ & 209 & 11,3 & - & & 209 & 8,5 \\
\hline $\mathrm{P}$ & $\begin{array}{l}\text { : Pamphlets, revues, journaux } \\
\text { illustrés, etc... }\end{array}$ & 64 & 3,4 & - & & 64 & 2,6 \\
\hline Q & $\begin{array}{l}\text { : Sciences, agriculture, méde- } \\
\text { cine, histoire naturelle, etc... }\end{array}$ & 117 & 6,3 & 46 & 7,7 & 163 & 6,6 \\
\hline R.S. & $\begin{array}{l}\text { : Revues, pamphlets, livres dépa- } \\
\text { reillés, etc... }\end{array}$ & 3 & 0,1 & - & & 3 & 0,1 \\
\hline $\mathrm{T}$ & $\begin{array}{l}\text { : Voyages, vies, mémoires, bio- } \\
\text { graphies, etc... }\end{array}$ & 115 & 6,2 & 89 & 14,8 & 204 & 8,3 \\
\hline TOTAL & & 1854 & 75,6 & 599 & 24,4 & 2453 & $100 \%$ \\
\hline
\end{tabular}

Comment classer ces titres? Selon la classification de l'Institut Canadien de 1870 et de 1876 ? Comment classera-t-on ensuite les titres des catalogues de telle ou telle autre bibliothèque de Montréal, du Québec? Et enfin, comment pourra-t-on comparer les catalogues de telle bibliothèque de Lyon, de Glasgow, de Boston ou de Toronto?

Ces considérations et d'autres nous ont conduit à proposer l'adoption de la classification de 1876 de Melvin Dewey pour résoudre ce 
problème méthodologique et permettre de faire débloquer, pour le $19 e$ siècle, la recherche comparée, au plan international, des contenus des bibliothèques ${ }^{3}$.

La présente étude constitue donc la première application systématique de cette classification, dont l'adoption est en partie légitimée, pour nous, par cette vérification empirique.

\section{La bibliothèque de l'Institut Canadien (1845-1880)}

Un rappel de l'histoire de la bibliothèque permettra de donner un aperçu de son dynamisme et des circonstances de préparation et de publication des Catalogues.

Lente à se constituer, la bibliothèque est de surcroît victime d'un incendie en février 1850. Elle fait son plein de volumes durant la décennie de 1850 (tableau 2) avant de connaître une nouvelle expansion durant la décennie de 1870 alors que la bibliothèque demeure la seule activité de l'Institut Canadien et que celui-ci a modifié sa constitution pour établir une bibliothèque davantage publique, jusqu'à sa fermeture en 1880 .

La «salle de nouvelles» (newsroom) fut sans doute l'endroit, à Montréal, où se fit la lecture la plus intensive de journaux et de périodiques: en 1857, l'Institut reçoit 126 titres différents - un sommet en provenance du Québec, du Canada, des États-Unis et d'Europe. Il est peu probable qu'on puisse mesurer exactement l'importance de cette salle de nouvelles; il est vraisemblable que son utilisation fut autant sinon plus importante que celle de la bibliothèque même.

L'emprunt des volumes de la bibliothèque suit la croissance de la collection: apogée durant la décennie de 1850 et durant celle de 1870 .

L'évolution des activités de l'Institut Canadien reflète les débats et déboires de l'institution. Même si l'Institut s'est politiquement et idéologiquement radicalisé depuis 1848, il n'a pas encore fait l'objet de condamnation au moment où paraît son premier Catalogue en 1852.

La situation s'envenime en 1858 et le litige entre l'Institut et Mgr Bourget porte sur les livres et sur la bibliothèque. Dans une lettre pastorale du 10 mars 1858 qui développe les idées de Pie IX à propos des «erreurs du temps» (encyclique et Syllabus, 1864), Mgr Bourget fait une première mise en garde générale aux «Instituts littéraires» et à leurs membres à propos des «livres contraires à la foi et aux moeurs» et des moyens à prendre pour «faire disparaître» et «purger» des bibliothèques «tous les livres impies ou obscènes qui s'y trouveraient». La littérature

3 Yvan Lamonde, «Une classification universelle pour l'étude des bibliothèques et de la librairie au 19e siècle» (à paraître en 1988). 


\section{TABLEAU 2}

Institut Canadien de Montréal, volumes, journaux, périodiques et circulation de volumes à la bibliothèque (1845-1879)

\begin{tabular}{|c|c|c|c|}
\hline & $\begin{array}{c}\text { VOLUMES } \\
\text { (sans les brochures) }\end{array}$ & $\begin{array}{l}\text { JOURNAUX ET } \\
\text { PÉRIODIQUES }\end{array}$ & CIRCULATION \\
\hline 1845 & 404 & - & 1300 \\
\hline 1846 & 496 & 15 & 350 \\
\hline 1847 & 885 & 22 & 480 \\
\hline 1848 & 1291 & 25 & 780 \\
\hline 1849 & - & - & - \\
\hline 1850 & 689 & 53 & - \\
\hline 1851 & 1500 & 60 & 2400 \\
\hline 1852 & 1962 & 48 & 3169 \\
\hline 1853 & 2701 & 66 & 3060 \\
\hline 1854 & 3177 & 83 & 4175 \\
\hline 1855 & 4006 & 110 & 3360 \\
\hline 1856 & 2974 & 118 & 3807 \\
\hline 1857 & 3043 & 126 & 3788 \\
\hline 1858 & 4270 & 117 & 3130 \\
\hline 1859 & 4541 & 113 & 2784 \\
\hline 1860 & 5144 & 87 & 477 \\
\hline 1861 & 5147 & 85 & 1803 \\
\hline 1862 & 5500 & - & - \\
\hline 1863 & 5586 & 68 & 1253 \\
\hline 1864 & 5646 & 78 & 1686 \\
\hline 1865 & - & 一 & 1200 \\
\hline 1866 & 6500 & 69 & - \\
\hline 1867 & 7424 & 69 & - \\
\hline 1868 & 7474 & 75 & - \\
\hline 1869 & 7848 & 75 & - \\
\hline 1870 & 8094 & - & 1500 \\
\hline 1871 & 6978 & - & 1383 \\
\hline 1872 & - & - & 1500 \\
\hline 1873 & 8987 & 67 & 3081 \\
\hline 1874 & 9241 & 70 & 4319 \\
\hline 1875 & 9513 & 70 & 5095 \\
\hline 1876 & 9871 & 63 & 4859 \\
\hline 1877 & 10191 & 59 & 6122 \\
\hline 1878 & 10471 & 59 & 6392 \\
\hline 1879 & 10657 & 53 & 5597 \\
\hline
\end{tabular}

Sources: Rapports annuels et Procès-verbaux (1855-1880) de l'Institut Canadien de Montréal. 
y trouve son compte: «Ne souscrivez à aucun journal capable, par ses doctrines anti-religieuses, ses romans passionnés et ses feuilletorıs immoraux, de gâter l'esprit et le coeur de vos enfants.»

La seconde lettre pastorale (30 avril 1858) de Mgr Bourget est plus explicite: «Sur l'Institut Canadien et contre les mauvais livres». A la suite de la réunion extraordinaire (13 avril) de l'Institut Canadien qui entend demeurer «seul compétent à juger de la moralité de sa bibliothèque», Mgr Bourget récuse cette prétention par la «certitude» que l'Église conduit «son troupeau dans les gras pâturages de la vérité» et qu'elle est «incontestablement investie du droit de régler l'administration de toutes les Bibliothèques du monde». Comparant ensuite le $\mathrm{Cata}$ logue imprimé (1852) de la bibliothèque de l'Institut Canadien et celui de l'Index, Mgr Bourget y trouve un «trop grand nombre» de titres à l'Index.

La voix épiscopale s'élève enfin dans une lettre pastorale du 31 mai 1858 contre la voix éditoriale, «contre les mauvais journaux», tout en proclamant la «sainte Alliance» de la religion et de la politique. Le message éditorial de la Lettre pastorale porte sur le journal «irreligieux» qui «combat l'existence de Dieu et de sa divine religion»; sur le journal «hérétique» qui «attaque la Sainte Église catholique»; sur le journal «impie» qui feint de respecter la religion pour mieux la ruiner; sur le journal libéral qui prétend «être libre dans ses opinions religieuses et politiques» et «qui voudrait que l'Église fût séparée de l'État»; sur le journal «immoral»

qui blesse la pudeur et les bonnes moeurs par ses feuilletons impurs, ses histoires d'amour, ses chansons lubriques, ses poésies lascives, ses romans impudiques, ses pièces théâtrales, enfin par tout ce qui enflamme les passions, ôte l'horreur du vice, inspire du dégoût pour la vertu, fait admirer des hommes qui ont été l'opprobre du genre humain, par leurs crimes monstrueux, qui sont préconisés comme de grandes vertus.

Des pourparlers entre des mandataires de l'Institut Canadien et Mgr Bourget d'octobre 1863 à novembre 1864 ne réussissent pas «à aplanir les difficultés». Supplique de l'Institut Canadien auprès de Pie IX en septembre 1865, mémoire de l'évêque de Montréal concernant l'Institut et tractations vaticanes aboutissent à une décision de Rome en juillet 1869: condamnation des doctrines de l'Institut Canadien contenues dans l'Annuaire de l'Institut pour 1868, du coup mis à l'Index, et défense pour les catholiques d'en faire partie. Le propos romain ne vise donc pas expressément la bibliothèque et son contenu, cheval de bataille de Mgr Bourget depuis dix ans.

La décision romaine contenue dans une Circulaire épiscopale du 16 juillet 1869 est lue aux prônes des églises du diocèse le 29 août. En octobre, l'Institut Canadien fait un second appel à Rome, où Gonzalve 
Doutre ira faire valoir le point de vue de l'Institut, fin décembre 1869début janvier 1870. Depuis la mort (18 novembre 1869) du typographe Joseph Guibord, membre de l'Institut Canadien, les péripéties du procès relatif au lieu de sépulture sont rapidement connues à Rome et motivent la décision finale (13 août 1870) du Saint-Office: «Enfin, faites savoir au dit Dessaulles que le St-Siège est persuadé que l'Institut Canadien, tant à cause des matières que l'on traite que des principes que l'on exprime qui méritent une entière réprobation, a renoncé au but primitif de sa fondation.» ${ }^{4}$

Le Catalogue de 1870 est publié dans ce contexte. L'opposition des membres de l'Institut Canadien, depuis 1858, à l'ingérence censoriale de l'évêque, tout comme l'inclusion au Catalogue de 1870 d'ouvrages à l'Index, donnent à penser que l'Institut a maintenu sa souveraineté. D'autant plus que les décisions romaines ne visaient pas la bibliothèque. Dès lors, le Catalogue de 1870 reflète bien la collection de l'Institut Canadien.

\section{Le Catalogue de 1852}

Sept ans après sa fondation, la bibliothèque de l'Institut Canadien de Montréal contient 646 titres dont $80 \%$ en langue française, $17 \%$ en langue anglaise et $3 \%$ en latin (tableau 3). Ces 646 titres se répartissent en 1409 volumes. Treize titres (20\%) de 10 volumes et plus totalisent 351 volumes, soit le quart du total des volumes ${ }^{5}$.

\section{TABLEAU 3}

Institut Canadien de Montréal, bibliothèque, nombre de volumes et de titres (catalogues de 1852, 1870-1876)

\begin{tabular}{|lc|c|c|}
\hline & $\mathbf{1 8 5 2}$ & \multicolumn{2}{|c|}{$\mathbf{1 8 7 0 - 1 8 7 6}$} \\
\hline Nombre de titres & 646 & $2453(1854+599)$ \\
Titres en français & 514 & $(79,7 \%)$ & $1942(1488+454)(79,1 \%)$ \\
Titres en anglais & $109(16,8 \%)$ & $463(320+143)(18,8 \%)$ \\
Titres en latin & $33(3,5 \%)$ & $42(40+2)(1,7 \%)$ \\
Titres autres & - & 6 & 6 \\
Nombre volumes & 1409 & $4884(3944+940)$ \\
\hline
\end{tabular}

4 La trame des événements et des documents est exposée dans Léon Pouliot, s.j., Monseigneur Bourget et son temps (Montréal, Bellarmin, 1976), IV: Affrontement avec l'Institut Canadien, 1858-1870), 160 p., bien que l'interprétation demeure discutable. Les trois lettres de 1858 se trouvent dans les Mandements des Évêques de Montréal, III: 356-375, 380-441 et, VI: 24-38 et les documents de la condamnation de 1869, VI: 38-50.

5 La Bibliothèque des romans (80 volumes), les Oeuvres complètes de Voltaire (70), le Dictionnaire de la conversation et de la lecture (66), Le Courrier des Etats-Unis (20), La Bibliothèque germanique (17), un Dictionnaire d'Histoire naturelle (15), les Oeuvres complètes de Mably (15) et de Molière (12), parmi les principaux titres. 
Le tiers des titres disponibles en 1852 est constitué d'ouvrages de littérature, et de littérature française majoritairement (tableau 4). Le roman (54 titres) domine avec Alexandre Dumas (10 titres), René de Chateaubriand (10), Honoré de Balzac (Oeuvres «complètes»), Eugène Sue (6), Paul de Kock (4) et Victor Hugo. Suivent en importance les essais (27 titres), dont certains titres de Mme de Staël, puis la poésie (19). À nouveau, le roman domine (25 des 37 titres) la littérature anglaise offerte aux membres et abonnés de la bibliothèque. Les curieux d'histoire littéraire pouvaient consulter ou emprunter le Cours de littérature de La Harpe, le Cours de littérature française du moyen-âge et le Cours de littérature française au XVIIIe siècle de Villemain, l'Histoire littéraire de Voltaire et l'Histoire des littératures espagnole et française d'Adolphe de Puibusque, un ami des intellectuels canadiens-français de l'époque ${ }^{6}$.

Le quart des titres du Catalogue de 1852 concerne l'histoire. À elles seules, la littérature et l'histoire constituent donc plus de la moitié de la bibliothèque de l'Institut Canadien en 1852. Amateurs d'histoire, journalistes, hommes politiques, conférenciers et essayistes trouvaient à la bibliothèque une collection d'histoire moderne où dominait l'histoire de la France post-révolutionnaire et contemporaine; Thiers, Histoire de la Révolution française (8 volumes) et Histoire du Consulat et de l'Empire (10), et Guizot, Histoire de la civilisation (5) et Moyens de gouvernement, y occupaient une place prépondérante devant Thierry, Dix ans d'études historiques, Louis Blanc, Histoire de Dix ans, ou Michelet, Le peuple. Ceux qui bénéficient alors du gouvernement responsable et ceux qui demandent le rappel de l'Union pouvaient bien connaître l'histoire de l'Angleterre (10 titres): histoires de Lingard, de Thierry, de Guizot, de Carrel, Biographie du Parlement d'Angleterre, Essai sur la Constitution de l'Angleterre. Les observateurs du destin polonais pouvaient lire des histoires de la Pologne ou de la Russie.

L'histoire de l'Amérique du Nord - une trentaine de titres que nous analyserons plus loin dans «la bibliothèque» étatsunienne et canadienne de l'Institut Canadien - devance en importance numérique l'histoire ancienne (14 titres). La biographie historique, attrayante pour les conférenciers contemporains, compte une vingtaine de titres. Quant au récit de voyage, il est essentiellement européen (14 titres), exceptionnellement nord-américain ( 2 titres).

Les ouvrages traitant de la société constituent le cinquième de la bibliothèque. Les membres de cette société «littéraire» politiquement

6 Pour une analyse plus détaillée, Y. Lamonde «Institution et association littéraires au Québec au 19e siècle: le cas de l'Institut Canadien de Montréal (1852-1876)» (à paraître dans Littératures, 1 (1988), Cahiers du Département de langue et littérature françaises de l'Université McGill). 
identifiée et radicalisée en 1848 et qui allaient connaître leurs plus grands succès électoraux en 1854 avaient accès à des ouvrages de science politique; Pierre Leroux s'y trouve avec des titres politiques et philosophiques, aux côtés de Montesquieu, L'esprit des lois, les Lettres persanes et des Oeuvres diverses, de Machiavel, Politiques, de De Lolme, Constitution d'Angleterre, de Lamennais, Le livre du peuple et d'auteurs canadiens-français: Louis-Antoine Dessaulles, membre de l'Institut à compter de 1855, Lectures sur l'annexion et Antoine GérinLajoie, conférencier à l'Institut, Catéchisme politique. Membres et abonnés disposent aussi de documents parlementaires canadiens, typiques d'ailleurs de la bibliothèque montréalaise et québécoise de l'époque.

À cette époque de débats sur la fin du protectionnisme anglais et sur la réciprocité avec les États-Unis et en ces années où libre-échange, production et distribution des richesses deviennent les thèmes fréquents de conférences publiques ${ }^{7}$, les ouvrages d'économie politique occupent un rayon important de la bibliothèque: Oeuvres complètes de Ricardo et de Malthus, Catéchisme d'économie politique, Cours complet d'économie politique et Traité de Jean-Baptiste Say, Economistes financiers du XVIIIe siècle, Doctrines des physiocrates et Mélanges d'économie politique de E. Daire, Richesse des nations d'Adam Smith, Histoire de l'économie politique de Blanqui, Élements d'économie politique de J. Garnier, pour ne citer que les plus connus. S'ajoutent à ces titres de «politique», des ouvrages de droit et des volumes relatifs à l'éducation.

La théologie et la religion (53 titres, 8,2\%) ne constituent pas le rayon important de l'Institut Canadien de Montréal. On pouvait néanmoins y trouver des ouvrages sur la Bible, sur la doctrine du christianisme et sur l'éloquence de la chaire, tels ceux de Bossuet et de Massillon. Lamennais figure en bonne place dans le Catalogue et la bibliothèque avec les Affaires de Rome, l'Esquisse d'une philosophie, les Paroles d'un croyant et le Livre du peuple déjà mentionné.

Les Lumières constituent l'esssentiel du rayon Philosophie (31 titres): Voltaire, Oeuvres complètes en 70 volumes, Rousseau, Confessions, Contrat social, l'Émile, la Nouvelle Héloïse, Diderot, Oeuvres choisies, Helvétius, De l'esprit, d'Holbach, Morale universelle, Volney, Les ruines cléricales, s'alignent à côté de Pierre Leroux, de Bentham, Oeuvres complètes, et de quelques titres d'éthique.

Dans les Sciences appliquées (30 titres), on trouvait surtout des ouvrages de médecine ( 20 titres), tout comme l'astronomie (6 titres) et les mathématiques (5 titres) constituaient l'essentiel des Sciences natu-

\footnotetext{
7 Yvan Lamonde, Gens de parole. Conférences publiques, essais et débats à l'Institut Canadien de Montréal (1845-1871) (à paraître en 1988).
} 
relles (25 titres). La Philologie et les Arts - on n'a pas encore reçu les dons du prince Napoléon - sont peu présents au Catalogue.

L'Institut Canadien possède ou reçoit, en février 1852, quarantedeux journaux et périodiques (catégories 50-59 de la classification de 1876 de Dewey). Ce sont d'abord des périodiques canadiens (24) et montréalais (15); ce sont ensuite des périodiques français (10): L'Atelier (1845), les Archives littéraires de l'Europe (1804-1805), l'Écho des feuilletons (1841-1843), le Magasin pittoresque (1843), le Magasin universel (1835-1836), Le National (1850-1851), la Revue française (1835), la Semaine de Paris (1849-1851) et la Sentinelle du peuple (1850); puis des périodiques de Grande-Bretagne: Blackwood's Magazine (11 volumes), The Mirror (1825), Dublin Magazine et The Enquirer, et des États-Unis: l'important Courrier des États-Unis (1845, 18481851), The North American (1839-1840), le Franco-américain de New York (1847) et Le Citoyen de Détroit (1851).

\section{La bibliothèque étatsunienne}

Si le Courrier des États-Unis approvisionnait - surtout en hiver - les journaux du Québec et assurait de façon exceptionnelle l'acheminement de la nouvelle en provenance de France, il faut préciser que ces anciens exilés de 1837-1838, ces tenants de l'annexionnisme et de la réciprocité, tout comme les voyageurs, avaient développé une conscience américaine dont l'historiographie n'a pas encore pris la mesure. Les Six lectures sur l'annexion du Canada aux États-unis d'un L.-A. Dessaulles, faites à l'Institut même et disponibles à la bibliothèque, témoignent des connaissances documentaires à propos des États-Unis. La bibliothèque de l'Institut permettait certaines de ces recherches: à côté d'Atala et de René de Chateaubriand, de Fennimore Cooper ou des Moeurs des Américains de Miss Trolloppe, on trouvait en français et en anglais De la démocratie en Amérique de Tocqueville - dont les Lectures sur l'annexion constitueront une «suite» au dire du préfacier Joseph Doutre - et le roman à thèse de Gustave de Beaumont, Marie ou l'Esclavage aux États-Unis. On y trouvait aussi dans la veine de Tocqueville, L'Europe et l'Amérique comparée, des Recherches sur les Etats-Unis, deux études de Poussin, Puissance américaine et L'union américaine. L'Histoire de l'Amérique de W. Robinson, une History of the United States, une Vie de Washington, les Mémoires de B. Franklin étaient disponibles avec d'autres ouvrages sur les États de New York ou du Wisconsin.

\section{La bibliothèque canadienne}

L'étude de la section canadienne de la bibliothèque de l'Institut Canadien pose dans son entièreté le problème de notre connaissance de l'affirmation d'une littérature nationale et de l'expression d'une pensée 
québécoise. La connaissance de la culture québécoise ne passe pas seulement par l'analyse de la production de romans, d'essais ou d'histoires; elle passe tout autant par l'étude de sa diffusion et de sa consommation réelle. Cette histoire d'une «littérature nationale» doit comprendre l'analyse de ses modes de formation, de ses premiers supports d'expression, des lieux collectifs de consommation, tout comme celle de ses formes de capitalisation symbolique et d'appropriation en vue de son développement.

À une décennie des premières publications «littéraires», que révèle, à propos de la culture de l'imprimé et de la littérature, le Catalogue de 1852 de la bibliothèque de l'Institut Canadien de Montréal? L'importance d'abord de cette «bibliothèque du peuple» qu'est la presse. Quarante-deux titres de périodiques, dont quinze montréalais ${ }^{8}$ sont disponibles dans la «salle de nouvelles» (news room) de l'Institut. Une expression politique et une représentation critique de soi s'y formulent de façon nouvelle, intensive.

Le débat public, qui façonne une opinion publique qui va en se polarisant, s'alimente à la vie parlementaire et législative. Le Catalogue énumère ces documents publics et parlementaires qui nourrissent les débats contemporains à l'Institut ou dans la presse sur l'émigration, sur l'éducation, sur l'agriculture, sur le commerce, sur les brevets, sur la tenure seigneuriale. L'imprimé à l'Institut Canadien en 1852 est un imprimé «public»: des «papiers» publics, des documents publics. Les «publications» indiquent tout simplement l'émergence d'une sphère publique.

Par la place qu'il fait à l'histoire canadienne, le Catalogue de 1852 témoigne que se superpose à cette conscience publique et à cette conscience politique une conscience historique nouvelle. L'Histoire $d u$ Canada de Michel Bibaud (3 exemplaires), celle de F.-X. Garneau (3 volumes) et celle de Christie voisinent la Biographie des Sagamos illustres du fils de Michel Bibaud, Maximilien et quelques autres ouvrages d'histoire.

Tout comme pour l'histoire, ce qui est disponible en littérature canadienne se trouve sur les rayons: les Épîtres et satires de Michel Bibaud, Les Fiancés de 1812 de Joseph Doutre, ci-devant figure centrale de l'Institut, les Révélations du crime ou Cambray et ses complices

$8 \quad$ Les plus importants: L'Avenir (1847-1851), La Minerve (1827-1837), les Mélanges religieux (1840-1846), Le Fantasque (1837-1840), le Journal d'Agriculture (1848-1851) en français et en anglais; d'anciens journaux: Le Canadien (1807-1808), La Gazette de Québec (1807-1811), Le Constitutionnel (1823-1824), Le Courrier de Québec (1807-1808). Puis les albums de La Minerve et de la Revue canadienne, L'Ami de la religion et de la patrie (1848), L'Artisan (1842), Le Coin du feu, L'Encyclopédie canadienne (1842-1843), Le Journal de Québec (1846), Le Ménestrel (1844), Le Moniteur canadien (1849), L'Ordre social (1850), La Quotidienne, le Canada Temperance Advocate (1849), le Répertoire national. 
de F.-R. Angers, le Répertoire national de James Huston, membre actif de l'Institut, sans oublier le scandaleux Awful Exposure of Maria Monk.

\section{Les catalogues de 1870 et de 1876}

Malgré l'incendie qui détruit la bibliothèque et les archives en février 1850 , le nombre de titres passe de 646 en 1852 à 1854 en 1870 et à 2453 en 1876 . Le nombre de titres a donc quadruplé et celui des volumes (1 409 à 4 884) plus que triplé.

Cinquante-sept titres (soit $23,2 \%$ du total des titres) de dix volumes et plus regroupent 1433 volumes, soit $29,3 \%$ du total de volumes des catalogues de 1870 et de $1876^{9}$. La répartition des titres selon la langue (tableau 3) demeure similaire, compte tenu d'une légère augmentation (2\%) du nombre de titres en anglais, plus nombreux en littérature, en histoire de la Grande-Bretagne et de l'Amérique du Nord.

La littérature et l'histoire qui constituaient un peu plus de la moitié de la bibliothèque en 1852 forment en 1870-1876 les deux tiers (67\%) du catalogue. Une seule différence notoire, à un quart de siècle de distance: l'augmentation $(+13 \%)$ de la littérature, c'est-à-dire du roman populaire, dans l'ensemble du catalogue. Les autres rubriques de la classification de 1876 de Dewey varient peu, de $-4 \%$ à 0\% (tableaux 4 et 5). Cette évolution doit peu par ailleurs au don de gravures et de volumes - comptes rendus des Académies de l'Institut, recueil de documents sur l'histoire de la France - fait par le prince Napoléon lors de son passage à Montréal et à l'Institut Canadien en $1861^{10}$.

La littérature française continue de dominer la section littérature grâce essentiellement au roman qui forme $90 \%$ de cette production littéraire française. Feuilletonnistes et romanciers populaires occupent une place centrale au Catalogue (tableau 6):

Ces 492 titres constituent près des deux tiers $(63 \%)$ de la totalité (780) des romans français. Chateaubriand, Lamartine, Hugo, Balzac, Musset figurent honorablement aussi aux Catalogues. Le théâtre (27 titres) et la poésie (24) viennent loin derrière le roman.

Quelques titres se sont ajoutés en histoire littéraire, en particulier le Cours de littérature de Lamartine dont le battage publicitaire s'est fait à l'Institut Canadien par la visite d'un ami de Lamartine, J.-B.

9 Quelques exemples: Bibliothèque des dames (107 volumes), Bibliothèque des romans (80), Oeuvres de Voltaire (70), Dictionnaire de la conversation (68), Encyclopédie de Diderot et d'Alembert (36), Encyclopédie et annuaire du XIXe siècle (34), Oeuvres de Condillac (33), Correspondance de Napoléon ler (32). L'auteur remercie Claudine Rochat qui, sous sa supervision, a classé les titres du Catalogue de 1870.

10 Philippe Sylvain, «La visite du prince Napoléon au Canada (1861)», Mémoires de la Société royale du Canada, 4e série, II (1964): 105-127. 
Desplace, et par des conférences publiques de ce dernier et de L.-A. Dessaulles $^{11}$.

La littérature anglaise, en langue originale ou en traduction, a connu une croissance remarquable depuis 1852, passant de 37 à 107 titres. Dickens, Thackeray, Defoe (Robinson Crusoe), Swift (Gulliver), Byron, Shelley, Milton, Macaulay étaient disponibles.

L'importance globale de l'Histoire demeure relativement la même: l'histoire française d'après 1789 domine toujours avec les mêmes historiens auxquels se sont ajoutés, en particulier, les ouvrages historiques de Lamartine, Histoire de la Restauration et Les Girondins, tous deux en huit volumes, l'Histoire de la Révolution française de 1848, en français et en anglais.

L'histoire de la Grande-Bretagne est mieux documentée en 18701876 qu'en 1852 (26 titres contre 10), tout comme celle de l'Italie où l'on disposait à propos de la question romaine et de l'unité italienne, en plus des journaux, de la Révolution de Rome en 1848 de A. Balleydier, de l'Histoire de la liberté en Italie de Sismondi et de la Question italienne du prince Napoléon.

Une cinquantaine de titres sur l'histoire de l'Amérique du Nord, et majoritairement sur l'histoire du Canada, se sont ajoutés à la bibliothèque étatsunienne et canadienne (81 titres), sur laquelle nous reviendrons.

L'importance relative accordée à la littérature de voyage, en particulier en Amérique du Nord, a quintuplé, passant de 16 titres en 1852 à 85 en 1876.

Le tiers résiduel des Catalogues de 1870 et de 1876 comprend par ordre d'importance (tableau 5): la Théologie (188 titres), la Sociologie (166), la Bibliographie (87), les Sciences appliquées (78), les Arts (51) et la Philologie (38).

La percée positiviste parmi les ouvrages de théologie et de religion est remarquable. Renan, Vie de Jésus, Études d'histoire religieuse, la Nouvelle symbolique, St-Paul, Les apôtres, Histoire des langues sémitiques, Edgar Quinet, Genre des religions, Jules Simon, Liberté de conscience, Religion, voisinent encore des ouvrages de controverse, en anglais surtout et des «comparaisons» entre le protestantisme et le catholicisme.

11 Philippe Sylvain, «Lamartine et les catholiques de France et du Canada», Revue d'histoire de l'Amérique française, 4,1 (juin 1950): 29-60; 4,2 (septembre 1950): 233-248): 4,3 (décembre 1950): 375-397; Séraphin Marion, «Lamartine et l'Institut Canadien de Montréal», Lettres canadiennes d'autrefois (Hull, Éditions Éclair, 1954), 8: 39-75. 


\section{TABLEAU 4}

Institut Canadien de Montréal, nombre de titres au Catalogue de 1852, selon la classification Dewey de 1876

\begin{tabular}{|c|c|c|c|c|c|c|c|}
\hline & & $\mathrm{N}$. & $\%$ & & & $\mathrm{~N}$. & $\%$ \\
\hline $\begin{array}{r}0 \\
10 \\
20 \\
30 \\
40 \\
50 \\
60 \\
70 \\
80 \\
90\end{array}$ & $\begin{array}{l}\text { Bibliographie } \\
\text { Bibliothéconomie } \\
\text { Encyclopédies générales } \\
\text { Polygraphie } \\
\text { Périodiques généraux } \\
\text { Organisations générales }\end{array}$ & 46 & 7,1 & $\begin{array}{l}500 \\
510 \\
520 \\
530 \\
540 \\
550 \\
560 \\
570 \\
580 \\
590\end{array}$ & $\begin{array}{l}\text { SCIENCES NATURELLES } \\
\text { Mathématiques } \\
\text { Astronomie } \\
\text { Physique } \\
\text { Chimie } \\
\text { Géologie } \\
\text { Paléontologie } \\
\text { Biologie } \\
\text { Botanique } \\
\text { Zoologie }\end{array}$ & $\begin{array}{r}25 \\
5 \\
6\end{array}$ & 3,9 \\
\hline $\begin{array}{l}100 \\
110 \\
120 \\
130 \\
140 \\
150 \\
160 \\
170 \\
180 \\
190\end{array}$ & $\begin{array}{l}\text { PHILOSOPHIE } \\
\text { Métaphysique } \\
\\
\text { Anthropologie } \\
\text { Écoles de psychologie } \\
\text { Psychologie } \\
\text { Logique } \\
\text { Morale } \\
\text { Philosophies anciennes } \\
\text { Philosophies modernes }\end{array}$ & 31 & 4,8 & $\begin{array}{l}600 \\
610 \\
620 \\
630 \\
640 \\
650 \\
660 \\
670 \\
680 \\
690\end{array}$ & $\begin{array}{l}\text { SCIENCES APPLIQUÉES } \\
\text { Médecine } \\
\text { Génie } \\
\text { Agriculture } \\
\text { Économie domestique } \\
\text { Affaires } \\
\text { Chimie appliquée } \\
\text { Industries } \\
\text { Assemblage/finition } \\
\text { Construction }\end{array}$ & $\begin{array}{l}30 \\
20\end{array}$ & 4,6 \\
\hline $\begin{array}{l}200 \\
210 \\
220 \\
230 \\
240 \\
250 \\
260 \\
270 \\
280 \\
290\end{array}$ & $\begin{array}{l}\text { THÉOLOGIE/Religion } \\
\text { Religion naturelle } \\
\text { Bible } \\
\text { Théologie dogmatique } \\
\text { Piété et dévotion } \\
\text { Prédication et pastorale } \\
\text { Institutions et missions } \\
\text { Histoire ecclésiastique } \\
\text { Confessions chrétiennes } \\
\text { Confessions non-chrétiennes }\end{array}$ & $\begin{array}{r}53 \\
10 \\
9 \\
9 \\
8\end{array}$ & 8,2 & $\begin{array}{l}700 \\
710 \\
720 \\
730 \\
740 \\
750 \\
760 \\
770 \\
780 \\
790\end{array}$ & $\begin{array}{l}\text { ARTS } \\
\text { Aménagement } \\
\text { Architecture } \\
\text { Sculpture } \\
\text { Dessin et arts décoratifs } \\
\text { Peinture } \\
\text { Gravure } \\
\text { Photographie } \\
\text { Musique } \\
\text { Récréation }\end{array}$ & 9 & 1,4 \\
\hline $\begin{array}{l}310 \\
320 \\
330 \\
340 \\
350 \\
360 \\
370 \\
380 \\
390\end{array}$ & $\begin{array}{l}\text { SOCIOLOGIE/ } \\
\text { Sciences sociales } \\
\text { Statistiques } \\
\text { Science politique } \\
\text { Économie politique } \\
\text { Droit } \\
\text { Administration } \\
\text { Associations et institutions } \\
\text { Éducation } \\
\text { Commerce et communication } \\
\text { Coutumes et costumes }\end{array}$ & $\begin{array}{l}29 \\
19 \\
13\end{array}$ & 11,3 & $\begin{array}{l}800 \\
810 \\
820 \\
830 \\
840 \\
850 \\
860 \\
870 \\
880 \\
890\end{array}$ & $\begin{array}{l}\text { LITTÉRATURE } \\
\text { Traités et collections } \\
\text { Anglaise } \\
\text { Allemande } \\
\text { Française } \\
\text { Italienne } \\
\text { Espagnole } \\
\text { Latine } \\
\text { Grecque } \\
\text { Autres littératures }\end{array}$ & $\begin{array}{r}37 \\
120\end{array}$ & 32,7 \\
\hline $\begin{array}{l}400 \\
410 \\
420 \\
430 \\
440 \\
450 \\
460 \\
470 \\
480 \\
490\end{array}$ & $\begin{array}{l}\text { PHILOLOGIE/Langage } \\
\text { Comparée } \\
\text { Anglaise } \\
\text { Allemande } \\
\text { Française } \\
\text { Italienne } \\
\text { Espagnole } \\
\text { Latine } \\
\text { Grecque } \\
\text { Autres langues }\end{array}$ & 11 & 1,7 & $\begin{array}{l}900 \\
910 \\
920 \\
930 \\
940 \\
950 \\
960 \\
970 \\
980 \\
990\end{array}$ & $\begin{array}{l}\text { HISTOIRE } \\
\text { Géographie et observations } \\
\text { Biographie } \\
\text { Histoire de l'Antiquité } \\
\text { Europe } \\
\text { Asie } \\
\text { Afrique } \\
\text { Amérique du Nord } \\
\text { Amérique du Sud } \\
\text { Océanie et régions polaires }\end{array}$ & $\begin{array}{r}157 \\
16 \\
23 \\
14 \\
58\end{array}$ & 24,3 \\
\hline 490 & & & & & TOTAL & 646 & \\
\hline
\end{tabular}


TABLEAU 5

Institut Canadien de Montréal, nombre de titres au Catalogue de 1870-1876, selon la classification Dewey de 1876

\begin{tabular}{|c|c|c|c|c|c|c|c|}
\hline \multirow{11}{*}{$\begin{array}{r}0 \\
10 \\
20 \\
30 \\
40 \\
50 \\
60 \\
70 \\
80 \\
90\end{array}$} & \multirow{11}{*}{$\begin{array}{l}\text { Bibliographie } \\
\text { Bibliothéconomie } \\
\text { Encyclopédies générales } \\
\text { Polygraphie } \\
\text { Périodiques généraux } \\
\text { Organisations générales }\end{array}$} & $\mathrm{N}$. & $\%$ & & & $\mathrm{~N}$. & $\%$ \\
\hline & & \multirow{5}{*}{100} & \multirow{10}{*}{4.1} & 500 & SCIENCES NATURELLES & 88 & 3,6 \\
\hline & & & & 510 & Mathématiques & 7 & \\
\hline & & & & 520 & Astronomie & \multirow{2}{*}{\multicolumn{2}{|c|}{8}} \\
\hline & & & & 530 & Physique & & \\
\hline & & & & 540 & Chimie & \multirow[t]{6}{*}{8} & \\
\hline & & \multirow{5}{*}{71} & & 550 & Géologie & & \\
\hline & & & & 560 & Paléontologie & & \\
\hline & & & & 570 & Biologie & & \\
\hline & & & & 580 & Botanique & & \\
\hline & & & & 590 & Zoologie & & \\
\hline \multirow[t]{2}{*}{100} & PHILOSOPHIE & \multirow[t]{9}{*}{87} & \multirow[t]{10}{*}{3,6} & 600 & SCIENCES APPLIQUÉES & \multirow{10}{*}{$\begin{array}{l}78 \\
16\end{array}$} & \multirow{10}{*}{3,2} \\
\hline & \multirow{2}{*}{ Métaphysique } & & & 610 & Médecine & & \\
\hline \multirow{8}{*}{$\begin{array}{l}110 \\
120 \\
130 \\
140 \\
150 \\
160 \\
170 \\
180 \\
190\end{array}$} & & & & $\begin{array}{l}610 \\
620\end{array}$ & $\begin{array}{l}\text { Medecine } \\
\text { Génie }\end{array}$ & & \\
\hline & Anthropologie & & & 630 & Agriculture & & \\
\hline & Écoles de psychologie & & & 640 & Economie domestique & & \\
\hline & Psychologie & & & 650 & Affaires & & \\
\hline & Logique & & & 660 & Chimie appliquée & & \\
\hline & Morale & & & 670 & Industries & & \\
\hline & Philosophies anciennes & & & 680 & Assemblage/finition & & \\
\hline & Philosophies modernes & 37 & & 690 & Construction & & \\
\hline 200 & THÉOLOGIE/Religion & 188 & \multirow{10}{*}{7,8} & 700 & ARTS & \multirow[t]{10}{*}{51} & 2,1 \\
\hline 210 & Religion naturelle & & & 710 & Aménagement & & \\
\hline 220 & Bible & 17 & & 720 & Architecture & & \\
\hline 230 & Théologie dogmatique & 41 & & 730 & Sculpture & & \\
\hline 240 & Piété et dévotion & 50 & & 740 & Dessin et arts décoratifs & & \\
\hline 250 & Prédication et pastorale & & & 750 & Peinture & & \\
\hline 260 & Institutions et missions & & & 760 & Gravure & & \\
\hline 270 & Histoire ecclésiastique & 18 & & 770 & Photographie & & \\
\hline 280 & Confessions chrétiennes & & & 780 & Musique & & \\
\hline 290 & Confessions non-chrétiennes & & & 790 & Récréation & & \\
\hline 300 & SOCIOLOGIE/ & & & 800 & LITTÉRATURE & & \\
\hline & Sciences sociales & 166 & 6,9 & & & 1099 & 45,6 \\
\hline 310 & Statistiques & & & 810 & Traités et collections & & \\
\hline 320 & Science politique & 51 & & 820 & Anglaise & 107 & \\
\hline 330 & Économie politique & 23 & & 830 & Allemande & & \\
\hline 340 & Droit & 56 & & 840 & Française & 875 & \\
\hline 350 & Administration & & & 850 & Italienne & & \\
\hline 360 & Associations et institutions & & & 860 & Espagnole & & \\
\hline 370 & Éducation & 11 & & 870 & Latine & 63 & \\
\hline 380 & Commerce et communication & & & 880 & Grecque & & \\
\hline 390 & Coutumes et costumes & & & 890 & Autres littératures & & \\
\hline 400 & PHILOLOGIE/Langage & 38 & 1,6 & 900 & HISTOIRE & 516 & 21,4 \\
\hline 410 & Comparée & & & 910 & Géographie et observations & 85 & \\
\hline 420 & Anglaise & & & 920 & Biographie & 66 & \\
\hline 430 & Allemande & & & 930 & Histoire de l'Antiquité & & \\
\hline 440 & Française & & & 940 & Europe & 186 & \\
\hline 450 & Italienne & & & 950 & Asie & & \\
\hline 460 & Espagnole & & & 960 & Afrique & & \\
\hline 470 & Latine & & & 970 & Amérique du Nord & 81 & \\
\hline 480 & Grecque & & & 980 & Amérique du Sud & & \\
\hline 490 & Autres langues & & & 990 & Océanie et régions polaires & & \\
\hline & & & & & TOTAL & $2411 *$ & \\
\hline 42 & & & & & cisés (soit $1.7 \%$ ) & & \\
\hline
\end{tabular}




\author{
TABLEAU 6 \\ Institut Canadien de Montréal, \\ romanciers et feuilletonnistes français aux Catalogues
}

$(1870,1876)(n=$ titres $)$

$\begin{array}{lr}\text { Alexandre Dumas } & 137 \\ \text { Émile Souvestre } & 47 \\ \text { Paul Féval } & 39 \\ \text { Gustave Aimard } & 38 \\ \text { George Sand } & 26 \\ \text { Ponson du Terrail } & 25 \\ \text { Frédéric Soulié } & 24 \\ \text { Henri Conscience } & 21 \\ \text { Henri Murger } & 21 \\ \text { Erkman-Chatriand } & 19 \\ \text { Eugène Sue } & 16 \\ \text { Jules Verne } & 15 \\ \text { Ernest Capendu } & 15 \\ \text { Louis Reybaud } & 15 \\ \text { Alphonse Karr } & 14 \\ \text { Paul de Kock } & 12 \\ \text { Xavier de Montepin } & 8\end{array}$

En Sociologie, les ouvrages de droit et de politique, principalement canadiens, ont surpassé ceux d'économie politique où n'apparaît comme nouveauté que les Essays on Political Economy de Bastiat.

Les ouvrages de chimie renouvellent les Sciences naturelles en 1870 et en 1876.

La Philosophie, riche en ouvrages du dix-huitième siècle, fait place à Auguste Comte, Positive Philosophy, à Victor Cousin, Histoire de la philosophie, à Locke, à des auteurs de «mental» et «d'experimental philosophies» (Paley, Parker, Upman) et à des philosophes sociaux: Pierre Leroux, De l'égalité, De l'humanité, par exemple, et Proudhon, Confessions d'un révolutionnaire, De la justice dans les révolutions.

Une nouveauté dans les Sciences appliquées: les ouvrages sur l'agriculture, en particulier aux États-Unis.

Les Arts, enfin, tiennent leur importance nouvelle d'ouvrages sur la photographie et de gravures reçues du prince Napoléon et qui permettront de constituer, pendant un certain temps, un modeste musée à l'Institut Canadien. 
La rubrique Bibliographie (divisions 0 à 99 de la classification) doit essentiellement son importance aux journaux et aux périodiques. Soixante et onze titres (contre 42 en 1852) sont présents aux Catalogues: ce sont surtout des journaux et périodiques canadiens (32) que nous analyserons plus loin, français (20), britanniques (10) et étatsuniens (9).

\section{La bibliothèque étatsunienne}

Les perspectives Nord-Sud ont changé depuis 1852; à la tentation annexionniste et aux espoirs de la réciprocité commerciale ont succédé des échanges touristiques plus fréquents et une attention privilégiée à un événement majeur: la guerre de Sécession.

La bibliothèque étatsunienne de l'Institut Canadien comprend toujours en 1876 des journaux et des périodiques américains, vraisemblablement plus nombreux d'ailleurs que ceux qui sont énumérés dans le Catalogue de 1870; on dispose du American Newspapers Directory de Rowell pour faire un choix qui inclut alors des journaux en langue française: Le Courrier des États-Unis depuis 1848, Le Messager francoaméricain depuis 1864, la Revue du nouveau monde de New York du journaliste et romancier Régis de Trobriand et des titres en langue anglaise: Farmers' Journal, Hunt's Merchant Magazine, Harper's Weekly depuis 1860.

Les relations de voyage aux États-Unis se sont multipliées, de même que les études: Le système pénitentiaire aux États-Unis de Tocqueville et de Beaumont, L'Europe et l'Amérique comparée de Drouin de Barley, les Considerations on United States de Buissot, les Commentaires sur la Constitution des États-Unis de P. Odent, à titre d'exemples. On dispose d'une bonne dizaine d'histoires générales des États-Unis en français (Laboulaye, Brancroft, W. Robertson) ou en anglais (R. Stewart, C. B. Taylor, Goodrich), de travaux sur l'histoire (O'Callaghan sur l'État de New York, Hoskins sur le Vermont, French et Gayarré sur la Louisiane) et sur l'agriculture de certains États. Sur la guerre civile, on dispose de l'American Conflict, 1860-1864 d'Horace Greely du New York Tribune qui fera une brève conférence à l'Institut Canadien en 1867, de La guerre américaine (1865) de Louis-Antoine Dessaulles qui en fit d'abord le sujet d'une série de conférences publiques à l'Institut.

Fréchette avait bien raison d'écrire en 1867:

Le Canada aurait pu conquérir sa place au milieu des littératures du vieux monde, si parmi ses enfants il s'était trouvé un écrivain capable d'initier, avant Fennimore Cooper, l'Europe à la grandiose nature de nos forêts, aux exploits légendaires de nos trappeurs et de nos voyageurs. Aujourd'hui quand bien même un talent 
aussi puissant que celui de l'auteur du Dernier des Mohicans, se révélerait parmi nous, ses oeuvres ne produiraient aucune sensation en Europe, car il aurait l'irréparable tort d'arriver le second, c'est-à-dire trop tard. ${ }^{12}$

Les oeuvres de Fenimore Cooper apparaissaient aux Catalogues de 1870 et de 1876: Bravo, Prairie, Red Rover et Corsaire rouge, Spy, Dernier des Mohicans, Ontario, Oeil de Faucon, Pionniers, Robinson Américain.

Cet attrait pour l'exotisme américain est aussi corroboré par l'importance aux catalogues des romans «américains» du romancier populaire français Gustave Aimard et par les initiatives «américaines» du Français Henri-Émile Chevalier qui vit à Montréal de 1853 à 1860, y publie des feuilletons et s'active dans le réseau libéral de l'Institut Canadien, du Pays, puis de La Patrie.

\section{La bibliothèque canadienne}

En 1852, la bibliothèque de l'Institut Canadien de Montréal offrait 38 titres d'ouvrages canadiens et 42 journaux canadiens (soit à l'exclusion des journaux, 5,9\% du total des titres) comparativement à 122 titres d'ouvrages et à 32 journaux canadiens $(5,1 \%)$ en $1870-1876$. Même proportion d'ouvrages donc, avec une baisse du nombre de journaux canadiens, vraisemblablement causée par une plus grande sélection idéologique.

En 1870-1876, trente-quatre de ces ouvrages canadiens (un peu plus du quart) sont de langue anglaise, dont 23 qui concernent le Canada en général. Y dominent de façon presque majoritaire $(41 \%)$ les ouvrages d'histoire (Annexe 1), les «histoires» du pays de Charlevoix, de Dussieux, de Bibaud, de Garneau, de Christie, de Brasseur, de Smith, de Parkman, de Turcotte; une dizaine de titres de récits ou de guides de voyage étaient aussi disponibles.

Dans cet Institut où les gens de droit assumaient un leadership évident et où la politique traversait de part en part l'association «littéraire», les ouvrages de droit ou relatifs à l'administration publique de même que les essais et pamphlets politiques accaparaient l'essentiel du rayon Sociologie.

À côté des répertoires et histoires (Huston, Lareau publié en 1874, La littérature canadienne de 1850 à 1860), le romanesque meublait surtout la bibliothèque littéraire canadienne-française (21 titres) de l'Institut: cinq titres d'H.-E. Chevalier, membre actif de l'Institut, Jacques et Marie de Napoléon Bourassa, Une de perdue, deux de trou-

12 Lettre à H.-R. Casgrain, 29 janvier 1867, cité dans Guildo Rousseau, L' image des ÉtatsUnis dans la littérature québécoise (l775-1930) (Sherbrooke, Éditions Naaman, 1981), 114. 
vées de Georges de Boucherville, Charles Guérin de P.-J.-O. Chauveau, Jean Rivard d'Antoine Gérin-Lajoie. Certains titres de romans importants ne s'y trouvaient pas: Les fiancés de 1812 (1844) de Joseph Doutre, un membre très actif de l'Institut, L'influence d' un livre (1837) de Gaspé fils et les Anciens Canadiens (1863) de Gaspé père, La terre paternelle (1871) de Patrice Lacombe, L'Intendant Bigot (1872) et le Chevalier de Mornac (1873) de Joseph Marmette, sans compter des romans comme Hélika (1872) de Charles Deguise, L'héritière d'un millionnaire (1867) de Charles Marcil, Le rebelle (1842) du Français newyorkais Trobriand aussi publié à Montréal, et deux titres traduits de Madame Leprohon, Armand Durand (1869) et le Manoir de Villerai (1861).

En théologie et en religion, deux titres dont Chiniquy, Le prêtre, la femme et le confessionnal. Quant aux Mandements des Évêques de Montréal: point au catalogue, à tout le moins!

Vingt-deux titres de journaux et périodiques s'étaient ajoutés à la liste de 1852, signe manifeste, d'après la liste (Annexe 1), que la guerre des mots était bien engagée entre Le Pays, La Lanterne et La Minerve, le Courrier du Canada, le Nouveau monde et le Journal de Québec. On connaissait, on lisait et on citait au texte ses ennemis!

\section{La circulation des volumes et l'Index}

Avec un échantillon de $50 \%$ des titres empruntés de la bibliothèque de l'Institut Canadien entre 1865 et 1875 et représentant 9393 prêts consentis à 1150 emprunteurs, Louis-Georges Harvey et Mark V. Olsen ont démontré l'important écart entre la collection et la circulation de la bibliothèque de l'Institut Canadien ${ }^{13}$. Leur analyse révèle un écart négatif, en regard de la collection, pour toutes les catégories de volumes (Histoire et Belles-Lettres: -10\% chacune), sauf pour le roman qui constitue $33 \%$ de leur corpus de la collection et $77 \%$ de celui de la circulation $(+44 \%)$. Leur analyse précise encore la popularité croissante du roman français: il constitue $66,5 \%$ de la circulation totale en $1865,85 \%$ en 1875. Les treize auteurs les plus empruntés - sept titres d'Alexandre Dumas (père) et trois d'Eugène Sue - constituent $8,5 \%$ de la circulation totale. À lui seul A. Dumas (père) totalise 20\% de tous les emprunts. En 1870 , ces dix titres de Dumas et Sue les plus empruntés sont tous à l'Index de l'Église catholique romaine.

De son point de vue l'évêque Bourget avait raison en 1858 d'affirmer la présence de livres à l'Index au Catalogue de 1852. On peut évaluer à environ $4 \%$ le nombre de titres et à $14 \%$ celui des volumes à

3 L.-G. Harvey et Mark Olsen, «La circulation de la bibliothèque...», loc. cit., tableaux 
l'Index au Catalogue de $1852^{14}$. Des vingt et un titres (sur 646) à l'Index, huit sont des ouvrages d'histoire, cinq des ouvrages de philosophie. Les romans populaires et leurs auteurs mis à l'Index surtout après 1862 sont pourtant déjà sur les rayons en 1852: A. Dumas avec neuf titres, Sue avec sept, Balzac avec ses «oeuvres complètes» ${ }^{15}$.

En 1870, 288 des 2283 titres (soit 12,6\%) de la collection de la bibliothèque de l'Institut Canadien apparaissent à l'Index ${ }^{16}$. Les romans constituent 9,5\% des titres à l'Index, lequel s'est grossi depuis le début de la décennie de 1860 de la condamnation romaine des romans et des romanciers populaires. En 1868, 50\% des titres en circulation sont à l'Index, selon Harvey et Olsen, et $47 \%$ de ce total est constitué de romans.

Toujours d'après Harvey et Olsen, c'est le groupe socio-occupationnel des petits commercants, des fonctionnaires et des commis qui emprunte le plus de titres entre 1865 et 1875 ( 2028 titres, soit $43,6 \%$ du total ${ }^{17}$; dans ce groupe, ce sont les commis ( 759 titres), les étudiants (281) et les douaniers (259) qui sont les principaux emprunteurs. Suit le groupe des marchands et des membres des professions libérales (1 361 titres, 29,4\%), et parmi eux les marchands (530 titres), les avocats (420) et les médecins (175) empruntent le plus. Dans le groupe des artisans (883 titres, 18,9\%), ce sont les policiers (176 titres), les tailleurs (107) et les bouchers (96) qui s'avèrent les principaux emprunteurs. Le groupe des ouvriers qualifiés (168 titres, $3,2 \%)$ et celui des journaliers (16 titres, 0,3\%) se partagent le reste des emprunts.

Petits commerçants, fonctionnaires, commis et artisans augmentent même leurs emprunts après la condamnation romaine de 1869 , alors que les marchands et les gens de professions libérales, plus sensibles aux pressions cléricales, selon Harvey et Olsen, diminuent les leurs. Soixante-douze pour cent de la consommation romanesque s'avère celle des artisans, 67,4\% celle des petits commerçants, des fonctionnaires et des commis.

14 L.-G. Harvey, Étude de la bibliothèque, 55-62. Cette évaluation est fondée sur l'analyse que Harvey fait des volumes du Catalogue de 1870 qui sont dans l'édition de 1853 de l'Index et sur notre analyse du Catalogue de 1852 selon le nombre de titres à l'Index.

15 De Dumas: Ange Pitou, Le Collier de la Reine, La dame de Monsoreau (3 exemplaires), George, Monte-Cristo (3), La reine Margot, Les trois mousquetaires (2), Vingt ans après (2), Le comte de Bragelonne; de Sue: La bonne aventure, Les enfants de l'amour, Histoire de la Marine française, Le juif errant (2), Martin l'enfant trouvé (2), Les mystères de Paris, Les sept péchés capitaux.

${ }_{16}$ Ce total de Harvey et Olsen est composé des titres du Catalogue de 1870 et de 700 titres en circulation de 1865 à 1870 n'apparaissant pas au Catalogue de 1870.

17 Évaluation basée sur $50 \%$ des 1150 emprunteurs dont la profession est connue et portant sur 4456 prêts, soit $47,4 \%$ de la totalité $(9393)$ des prêts. 


\section{Conclusion}

Au plan méthodologique, la classification Dewey de 1876 s'est révélé un bon outil d'analyse pour mettre en valeur les richesses du corpus classé. Ce n'est pas une mince affaire, si on se rappelle que les historiens de l'imprimé ne disposaient pas d'un système de classification pour le 19e siècle. Dorénavant la comparaison entre deux bibliothèques d'une même ville, de deux provinces ou de deux pays est possible.

Des archives d'une richesse plutôt exceptionnelle ont aussi permis l'analyse de la collection et celle de la circulation de la bibliothèque de l'Institut Canadien. L'offre et la demande ont certes différé, mais le pattern de l'une et de l'autre ne différait pas au départ: le roman par exemple était dès 1852 majoritaire dans une littérature française ellemême dominante au Catalogue. Ajoutons, pour comprendre ce phénomène, la croissance «naturelle» de popularité du genre durant la seconde moitié du dix-neuvième siècle. Il faut enfin distinguer entre ce qu'on peut appeler la lecture de divertissement et de détente (le roman, par exemple) et la lecture de travail ou de recherche. Cette distinction renvoie aux usages variés d'une bibliothèque de collectivité: emprunt, mais aussi consultation sur place. L'importance cruciale de la «salle de nouvelles» dans une bibliothèque du dix-neuvième siècle ( $\mathrm{y}$ compris la lecture des feuilletons romanesques) sera sans cesse le rappel de la signification de la lecture sur place.

Qu'offrait donc à lire chez soi et sur place la bibliothèque de l'Institut Canadien sur un quart de siècle? D'abord et avant tout de la littérature et de l'histoire. De la littérature française, du roman, et ce de plus en plus de 1852 à 1876. De l'histoire moderne, française, postrévolutionnaire avec une évidente curiosité pour le personnage et l'époque de Napoléon; aux ouvrages de Thiers et de Guizot en particulier allaient s'ajouter les travaux historiques de Lamartine. Histoire française, mais aussi anglaise dans un pays qui modifie certes son lien colonial mais en le maintenant. Histoire de France et de Grande-Bretagne, histoire aussi des pays qui connaissent la montée du libéralisme et du nationalisme: la Pologne, l'Italie. Ce pluralisme intellectuel se double d'un pluralisme réel ou rêvé: la popularité croissante de la littérature de voyage en témoigne.

Le libéralisme de l'Institut s'alimentait aussi à sa bibliothèque, dans les livres de philosophie politique et, de façon méconnue jusqu'à maintenant, dans des ouvrages d'économie politique, abondamment cités dans de nombreuses conférences publiques à l'Institut même sur le sujet. Ce libéralisme pouvait puiser encore chez les philosophes des Lumières - et même chez Pierre Leroux et Joseph Proudhon - son argumentaire. Le versant anti-clérical de ce libéralisme évolua dans ses 
sources jusqu'à faire place au courant positiviste d'analyse du phénomène religieux.

La proximité géographique, la conjoncture économique de la Réciprocité et politique de la guerre de Sécession tout comme le modèle de la Grande République pour les libéraux montréalais incitent à mieux identifier la bibliothèque étatsunienne de l'Institut Canadien: l'actualité, l'histoire et la culture des États-Unis y sont documentées par la presse, par des ouvrages historiques et par une littérature exotique à la Cooper.

Un volume sur vingt que la bibliothèque de l'Institut Canadien offrait à lire était canadien, et ce aussi bien en 1852 qu'en 1876. Autant par sa forme que par ses contenus, l'association culturelle du dix-neuvième siècle constitue un signe de l'émergence d'une sphère publique, d'une vie publique. La bibliothèque de ce type d'institution ne fait qu'enrichir cette dimension: la presse, «la bibliothèque pour tous» et les documents parlementaires canadiens développent et alimentent l'opinion publique, la conscience politique. S'y superpose une conscience historique nouvelle qui dans la bibliothèque fait une place prépondérante aux histoires du pays. Le roman et les compilations de littérature «canadienne» témoignent enfin de l'émergence d'une symbolique et d'un imaginaire nouveaux.

$\mathrm{Au}$ terme de cette analyse du contenu de la bibliothèque de l'Institut Canadien, il faut revoir la signification de la condamnation de l'Institut. Il y a d'abord lieu de distinguer les condamnations: celle de Mgr Bourget focalisée sur la bibliothèque, les livres et les journaux et celle de Rome, plus générale, centrée sur la doctrine. Comme si l'évêque de Montréal avait été très sensible aux moyens, au médium et à l'émergence d'un nouveau discours public.

Il faut ensuite comprendre comment et pourquoi les condamnations de Mgr Bourget visaient plus la doctrine que la moralité alors que la collection et surtout la circulation étaient dans un rapport inverse. Il est certes question du caractère «immmoral» de certains titres dans les condamnations de l'évêque et les «romans passionnés» y sont mentionnés. Mais il y est beaucoup plus question du caractère doctrinal de la bibliothèque («irreligieux», «hérétique», «impie», «libéral»). Cette position renvoie bien sûr à l'antagonisme entre l'Eglise et la bourgeoisie libérale plus radicale: c'est une lutte de pouvoir social et idéologique. Elle renvoie aussi - côté verso - au rapport à la fois entre les libéraux et les couches populaires et entre l'Église et ces mêmes couches populaires.

Les libéraux de l'Institut Canadien ont tout de même fait place au roman dans leur bibliothèque; il faut bien comprendre qu'ils ont vu dans ce roman un possible travail idéologique convergent avec le leur. En ce 
sens, il faudra bien un jour qu'un littéraire dégage la signification idéologique de ce corpus de romans et de romanciers populaires... Divertissement romanesque et libéralisme sont-ils à ce point incompatibles comme le suggèrent Olsen et Harvey?

D'autre part, si l'Église pouvait condamner, montrer du doigt et excommunier quelques avocats, médecins ou étudiants lecteurs de Lamennais, Voltaire ou Renan, pouvait-elle s'attaquer à une forme culturelle nouvelle et aussi populaire que le roman? L'Église savait-elle faire la part des choses lorsque la doctrine était distillée dans le divertissement, lorsque le pernicieux se joignait à l'agréable, lorsque le roman s'offrait par tranche dans une presse à grand tirage? Les «papiers publics» devenus presse «populaire» défiaient dorénavant, tant par leur forme que par leur message populaire, le pouvoir temporel de l'Église. La question sociale émergeait dans la culture; elle allait bientôt prendre la forme d'une victoire des dirigeants du parc Sohmer pour faire légaliser (1893) l'ouverture du parc et la vente de bière le dimanche; elle allait marquer au coin le combat d'un Ernest Ouimet pour maintenir l'ouverture des salles de «vues animées» le dimanche. «L'immoralité» renvoyait à une autre morale sociale, à une nouvelle morale.

Il faut conclure enfin sur le destin de l'Institut Canadien. Il fut certes condamné et disparut en 1880 de la vie culturelle montréalaise. Mais les libéraux résistèrent: par leur résolution d'avril 1858, malgré les 138 démissions, avec les démarches auprès de l'évêque et les appels à Rome. Et malgré les condamnations locale et romaine, le pourcentage des titres à l'Index triple de 1852 à 1876 , passant de $4 \%$ à $12 \%$. Et surtout, le citoyen ordinaire qui lisait et empruntait des livres à l'Institut, lisait de plus en plus des romans, des romans à l'Index. En un sens, Dessaulles, Doutre et quelques autres finirent par se taire; mais on continua à lire...

\author{
ANNEXE 1 \\ Institut Canadien de Montréal, \\ catalogue reconstitué de la bibliothèque canadienne (1852-1876)
}

BIBLIOGRAPHIE (0-99): 3

Annuaire de l'Institut Canadien de Montréal de 1866 à 1870

Institut Canadien, 1852-1855

Catalogue of Mechanics' Institute Library

Journaux et périodiques

- Déjà au Catalogue de 1852:

Canada Temperance Advocate (1859)

Courrier de Québec (1806)

Le Canadien (1806)

L'Encyclopédie canadienne (1842)

Le Fantasque (1837-1840) 
Le Journal d'agriculture (1851, 1850)

L'Ordre social (1850)

Mélanges religieux (1841-

La Minerve (1826-

Le Canadien (1842- )

- Nouveaux:

La Bibliothèque canadienne (1825)

The Canadian Agriculturalist (1849)

Courrier du Canada (1856-1866)

Le Foyer canadien (1864)

Le Journal de Québec (1853-1870)

La Lanterne

The Ladies' Museum (1830)

L'Abeille canadienne (1818)

L'Agriculteur (1860-1861)

Le Magasin du Bas-Canada (1832)

The Montreal Herald (1861-

Nouveau monde (1867- )

L'Observateur

Le Pays (1852-

La Ruche littéraire (1853)

La Revue canadienne (1864)

La Semaine (1852)

Le Semeur (1842-1845)

La Semaine (1864)

Les Soirées canadiennes (1861)

\section{THÉOLOGIE (200-299): 2}

Rev. J. D. Borthwick, Harps of Canaan

Rev. Chiniquy, Le prêtre, la femme et le confessionnal

SOCIOLOGIE (300-399): 38

Statistique (310-319): 2

Recensement du Canada, 1870-71

Census of the Canadas, 1851-52

\section{Politique (320-329): 15}

Débats sur la Confédération de l' Amérique du Nord, 1865

Journaux des Assemblées législatives du Canada (avec Appendices)

J. E. Alexander, Canada as it was and may be

J.-C. Taché, Esquisses sur le Canada

H. Langevin, Essai sur le Canada

[Buies], Lettres sur le Canada

Politique canadienne, pamphlets

Rapport sur le gouvernement civil du Canada, 1829

Canadian Politics, pamphlets and reports

Settlers' Guide 
Conseiller du peuple

A. Achintre, Portraits et dossiers parlementaires

H. Morgan, Canadian Parliamentary Companion

Pamphlets divers sur le Canada

Proceedings of the House of Assembly, Lower Canada, 1815

Droit (340-349) et administration (350-359): 20

Actes des municipalités et des chemins, 1835

Actes et ordonnances du Bas-Canada, 1843

Actes relatifs aux pouvoirs des Juges de Paix, 1853

E. Lefebvre de Bellefeuille, Code civil du Bas-Canada

Ch. Glackmeyer, Charte et règlements de la cité de Montréal, 1865 Ordonnances,

Bas-Canada, 1777

G. Doutre, Procédure civile

Refus de sépulture (Affaire Guibord)

Statuts du Canada

H. Desrivières Beaubien, Traité sur les lois civiles du Bas-Canada

Règlements de police en force dans la cité de Montréal, 1843

Bibaud jeune, Commentaires sur les lois du Bas Canada

J. Crémazie, Lois criminelles anglaises

Doutre et Lareau, Droit civil canadien

Affaire Guibord (factum)

Affaire Guibord (plaidoiries)

Guibord case in Privy council

Guibord case, a History of the case

Rapports annuels de la corporation de Montréal

Commerce et communications (380-389): 1

Règles du chemin à rails du St-Laurent et de l'Atlantique

PHILOLOGIE (400-499): 1

Lacombe, Dictionnaire de langue Crise

\section{SCIENCES NATURELLES (500-599): 4}

J.-B. Meilleur, Cours abrégé de leçons de chimie

A. Heatherington, Gold Fields of Nova Scotia

Abbé Provancher, La Flore canadienne

Le Naturaliste canadien

\section{ARTS UTILES (600-699): 1}

Transactions of Board of Agriculture of Upper Canada, 1855

BEAUX-ARTS (700-799): 2

W. Notman, Photographic Selections

Plans d'un pont suspendu à Québec

\section{LITTÉRATURE (840-849): 21}

Henry Scadding, Canada and Oxford Longfellows, Poems 
J. Huston, Légendes canadiennes

Mrs. Leprohon, Antoinette de Mirecourt

J. Huston, Répertoire national

Littérature canadienne de 1850 à 1860

Napoléon Bourassa, Jacques et Marie

G. de Boucherville, Une de perdue deux de trouvées

P.-J.-O. Chauveau, Charles Guérin

H.-E. Chevalier, La huronne

Dernier Iroquois

Nez percés

Pieds noirs

Poignet d'acier

G. B. Chapin, Tales of the St Lawrence

A.-G. Lajoie, Jean Rivard

J. Marmette, François de Bienville

A. Buies, Chroniques, humeurs et caprices

M. Bibaud, Épîtres, satires, chansons, épigrammes

E. Lareau, Histoire de la littérature canadienne

$\mathrm{H}$. Larue, Mélanges historiques, littéraires, etc...

\section{HISTOIRE (900-999): 50}

Géographie et observations (910-919): 17

Division des comtés

Maps of Canada

Plans of Various Lakes and Rivers, 1853-1856

Rapport d'exploration entre le Lac Supérieur et la Rivière Rouge

L. Gingras, L'orient

de Basterot, De Québec à Lima

Rambles among the blue noses, a tour through New Brunswick and Nova Scotia

Travellers guide to the Hudson River ... and Quebec, 1864

Isaac Weld, Voyage au Canada, 1795

G. Franchère, fils, Voyage à la côte du Nord-Ouest, 1810

G. Franchère, Voyage to the North-West Coast of America

$\mathrm{G}$. Benson, Vie et voyage de Christophe Colomb

H. Lacroix, Canadian Guide

Abbé Provancher, Carte portative du Canada

J. Bouchette, Topographie du Canada

J.-M. LeMoine, Album du touriste

Géographie du Canada

Biographie (920-929): 4

Max. Bibaud, Biographie des Sagamos Illustres

Dictionnaire historique des hommes illustres du Canada

Panthéon canadien

Notice biographique sur François de Laval

\section{Histoire moderne (972): 29}

L. Dussieux, Canada sous la Domination française

D. Dainville [pseud.], Beautés de l'histoire du Canada 
R. H. Bonnycastle, Canada and the Canadians

Eighty Years of Progress of British North America

L.-A. Dessaulles, Guerre américaine

M. Bibaud, Histoire du Canada sous la domination française

F.-X. Garneau, Histoire du Canada depuis la découverte jusqu'à nos jours

Abbé Brasseur, Histoire du Canada, de son Église et de ses missions

Robert Christie, History of Lower Canada

de Charlevoix, Histoire de la Nouvelle-France

Chs. Lindsay, Life and Times of Wm MacKenzie

Mélanges sur le Canada

Quatre-vingt-douze résolutions, 24 fév. 1834

Souvenirs historiques sur le Canada

Life of Charles Lord Sydenham

Précis touchant la colonie de Lord Selkirk sur la Rivière Rouge, 1818 Bressany, Rela-

tion abrégée de quelques missions des pères de la Compagnie de Jésus

F.-B. Singer, Souvenir d' un exilé canadien

T.-A. Chandonnet, Notre-Dame-des-Canadiens

S. Drapeau, Colonisation du Bas-Canada de 1851 à 1861

C. Horetzki, Canada on the Pacific

$\mathrm{H}$. Kirke, Conquest of Canada

F. Parkman, Discovery of the Great-West

Jesuits in North America

Old Regime in Canada

France in the New World

J. Roy, History of Canada

Smith, Canada, Past, Present and Future

L.-P. Turcotte, Le Canada sous l'Union 